\title{
Global optimization of magnetic structure analysis by semidefinite relaxation method
}

\author{
R. Oishi-Tomiyasu ${ }^{1 *}$, K. Tomiyasu², M. Hagihara ${ }^{3}$ \\ ${ }^{I}$ Institute of Mathematics for Industry (IMI), Kyushu University, 744 Moto'oka, Nishi-Ward, Fukuoka, Japan, \\ ${ }^{2}$ NISSAN ARC Ltd., 1 Natsushima-cho, Yokosuka, Kanagawa, Japan, \\ ${ }^{3}$ High Energy Accelerator Research Organization, 203-1 Shirakata, Tokai, Naka, Ibaraki, Japan \\ tomiyasu@imi.kyushu-u.ac.jp
}

The local minima of optimized non-linear functions are a reason for ambiguity of the obtained solutions in crystal structure analysis and magnetic structure analysis (Figure 1). In this study [1], semidefinite programming relaxation (SDR) was applied for the first time to the determination of magnetic structure. Use of SDR allows us the following things (i) completing the global optimization procedure in a very short time (less than several seconds in many cases), (ii) judging whether the obtained solution is truly global one, there are multiple good candidates, or the irreducible representations considered are wrong with $100 \%$ probability. The solid foundation is provided by the duality theorem for convex optimization problems (Figure 2).

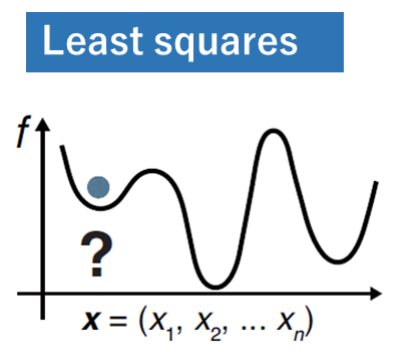

Figure 1. Local minimums of the optimized functions

\section{SDR}
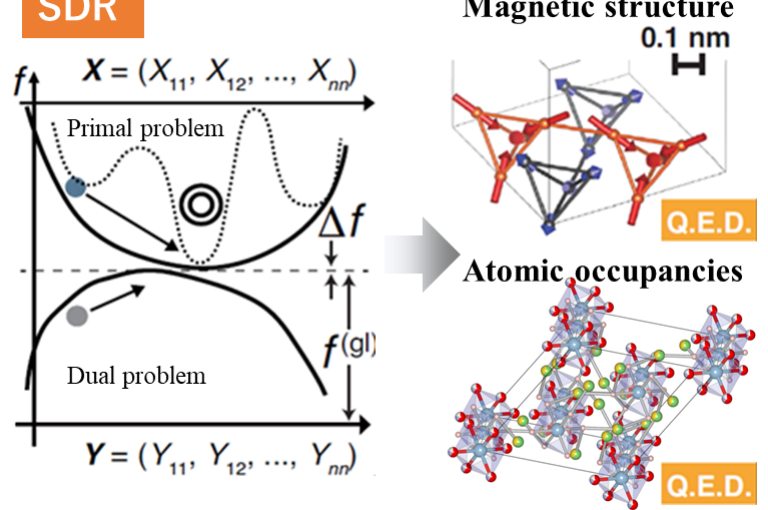

Figure 3. Example for almost periodic atomic coordinates

Figure 2. Global optimization by semidefinite programming relaxation (SDR)

In general, the global optimization of SDR is applicable for estimating not only magnetic moment vectors but also atomic occupancies at a fixed set of coordinates $\mathbf{x}_{1}, \ldots, \mathbf{x}_{m}$ from the absolute values of structure factors. Therefore, the method can be also used to judge if $x_{\mathrm{i}}$ is an atomic site or a void.

In many cases, this problem has a unique minimum solution. In some cases, there are a few distinct solutions, all of which can be constructed from the output of SDR. In a very few cases, the existence of multiple solutions is suggested by the SDR result. This often occurs when the atomic coordinates $\mathbf{x}_{1}, \ldots, \mathbf{x}_{m}$ in the unit cell are periodic or almost completely periodic as in Figure $\mathbf{3}$. If the symmetry of the atomic coordinates is considered, the existence of such multiple solutions can be largely eliminated.

As a result, SDR can provide a numerical answer to the classical problem of the uniqueness of solutions in crystal structure analysis [2]. Global optimization of the SDR method is now being implemented into Z-Rietveld software [3] distributed for users of J-PARC (Japan Proton Accelerator Research Complex).

[1] K. Tomiyasu, R. Oishi-Tomiyasu (mathematician \& one of corresponding authors), M. Matsuda and K. Matsuhira, Scientific Reports 8:16228 (2018).

[2] P. ENGEL, Comput. Math. Applic. 16 (1988), 425--436.

[3] https://z-code.kek.jp/zrg/

Keywords: Global optimization, Magnetic structure analysis, Rietveld software, Semidefinite relaxation (SDR)

This study was financially supported by JST PRESTO (grant No. JPMJPR14E6), JST JST-Mirai Program (grant No. JPMJMI18GD), and JSPS KAKENHI (JP17H06137 and JP18K03503). .

Acta Cryst. (2021), A77, C180 